\title{
BERNSTEIN INEQUALITIES FOR A CLASS OF RANDOM VARIABLES
}

\author{
M. SCHMUCKENSCHLAEGER
}

(Communicated by William J. Davis)

ABSTRACT. We prove a deviation inequality for sums of i.i.d. random variables.

Under the condition of the existence of an exponential moment, the Bernstein inequalities give an upper bound for the measure of the deviation of the arithmetic mean for independent identically distributed random variables with expectation zero. (For a precise formulation see [H] or [P]; for an application see, for example, [BLM].) We consider the weaker assumption

$$
\mathbf{E} \exp \left(X^{\alpha}\right)<\infty \text { for some } \alpha \in(0,1)
$$

and a nonnegative random variable $X$ and show for sufficiently large $t$, i.e., for $t>t_{0}$, where $t_{0}$ only depends on $\alpha$ and $\mathbf{E} X$, that

$$
\mathbf{P}\left(\frac{1}{n} \sum_{j=1}^{n} X_{j}-\mathbf{E} X>t \mathbf{E} X\right) \leq \exp -\left(c(\alpha)(n t \mathbf{E} X)^{\alpha}\right) .
$$

The method of proof follows a paper by Schechtman and Zinn [SZ]. The following lemma is elementary.

Lemma. (i) If $j \leq N:=\left[\frac{n+1}{2}\right]$ then $\log \left(\begin{array}{c}n \\ j\end{array}\right) \leq 2 j \log \left(\frac{n}{j}\right)$.

(ii) $\sum_{j=1}^{\infty} j^{-1 / \alpha} \leq(1-\alpha)^{-1} \forall 0<\alpha<1$.

(iii) $\sum_{j=1}^{N}\left(\log \left(\frac{n c}{j}\right)\right)^{1 / \alpha} \leq n c \Gamma\left(1+\frac{1}{\alpha}\right), c \geq 1$.

Proof. (i) is an immediate consequence of Stirling's formula and the fact that the function

$$
x \mapsto x \log \left(\frac{a}{x}\right)-(a-x) \log \left(\frac{a}{a-x}\right)
$$

is nondecreasing on $\left(\frac{a}{2}, a\right)$ and vanishes at $\frac{a}{2}$. For $j=\left[\frac{n+1}{2}\right]$ the inequality can be checked directly.

Received by the editors March 30, 1991.

1991 Mathematics Subject Classification. Primary 60E15; Secondary 60F10. 
(iii) is proved by replacing the sum by an integral:

$$
\begin{aligned}
\sum_{j=1}^{N} \log ^{1 / \alpha}\left(\frac{c n}{j}\right) & \leq \log ^{1 / \alpha}(c n)+\int_{1}^{N} \log ^{1 / \alpha}\left(\frac{c n}{t}\right) d t \\
& =\log ^{1 / \alpha}(c n)+c n \int_{\log (c n / N)}^{\log (c n)} x^{1 / \alpha} \exp (-x) d x \\
& <\log ^{1 / \alpha}(c n)+c n \Gamma\left(1+\frac{1}{\alpha}\right)-c n \int_{\log (c n)}^{\infty} x^{1 / \alpha} \exp (-x) d x \\
& \leq \log ^{1 / \alpha}(c n)+c n \Gamma\left(1+\frac{1}{\alpha}\right)-c n \frac{1}{c n} \log ^{1 / \alpha}(c n) \\
& =c n \Gamma\left(1+\frac{1}{\alpha}\right) .
\end{aligned}
$$

Let $0<\alpha<1$, and let $X$ be a nonnegative random variable such that

$$
\operatorname{E} \exp \left(X^{\alpha}\right)=A<\infty \text {. }
$$

Let $\left(X_{j}\right)_{j=1}^{n}$ be independent copies of $X$, and let $\left(X_{j}^{*}\right)$ be the nonincreasing rearrangement of $\left(X_{j}\right)$. Then for all $t>0$,

$$
\begin{aligned}
\mathbf{P}\left(\frac{1}{n} \sum_{j \leq n} X_{j}>t\right) & =\mathbf{P}\left(\sum_{j \leq n} X_{j}^{*}>n t\right) \\
& \leq \mathbf{P}\left(\sum_{j \leq N} X_{j}^{*}>\frac{1}{2} n t\right)+\mathbf{P}\left(\sum_{j>N} X_{j}^{*}>\frac{1}{2} n t\right) \\
& \leq 2 \mathbf{P}\left(\sum_{j \leq N} X_{j}^{*}>\frac{1}{2}(1+t) n E\right)
\end{aligned}
$$

where $N=[(n+1) / 2]$. Choose $p_{j}>0$ in such a way that $\sum_{j \leq N} p_{j} \leq 1$. Then

$$
\mathbf{P}\left(\sum_{j \leq N} X_{j}^{*}>\frac{1}{2} n t\right) \leq \sum_{j \leq N} \mathbf{P}\left(X_{j}^{*}>\frac{1}{2} p_{j} n t\right) .
$$

Since for all $s>0$

$$
\left[X_{j}^{*}>s\right]=\bigcup_{A(j)}\left\{\bigcap_{k \in A(j)}\left[X_{k}>s\right]: A(j) \subseteq\{1, \ldots, n\},|A(j)|=j\right\}
$$

and

$$
\mathbf{P}(X>s) \leq A \exp \left(-s^{\alpha}\right),
$$

by the lemma above we get

$$
\begin{aligned}
\mathbf{P}\left(X_{j}^{*}>s\right) & \leq A^{j}\left(\begin{array}{l}
n \\
j
\end{array}\right) \exp \left(-j s^{\alpha}\right) \\
& \leq \exp \left(j\left(\log A+2 \log \left(\frac{n}{j}\right)-s^{\alpha}\right)\right),
\end{aligned}
$$




$$
\mathbf{P}\left(\sum_{j \leq N} X_{j}^{*}>\frac{1}{2} n t\right) \leq \sum_{j \leq N} \exp \left(j\left(\log A+2 \log \left(\frac{n}{j}\right)-\left(\frac{1}{2} p_{j} n t\right)^{\alpha}\right)\right) .
$$

We now choose the $p_{j}$ so that we have the inequality

$$
j\left(\log A+2 \log \left(\frac{n}{j}\right)-\left(\frac{p_{j}}{2} n t\right)^{\alpha}\right) \leq-(c n t)^{\alpha}
$$

for some constant $c$, i.e.,

$$
\begin{aligned}
p_{j} & =\left((2 c)^{\alpha} \frac{1}{j}+\left(\frac{2}{n t}\right)^{\alpha}\left(\log A+2 \log \left(\frac{n}{j}\right)\right)\right)^{1 / \alpha} \\
& \leq 2^{1 / \alpha-1}\left((2 c) \frac{1}{j^{1 / \alpha}}+\frac{2}{n t}\left(\log A+2 \log \left(\frac{n}{j}\right)\right)^{1 / \alpha}\right) \\
& =2^{1 / \alpha} c j^{-1 / \alpha}+\frac{2^{2 / \alpha}}{n t} \log ^{1 / \alpha}\left(\frac{n \sqrt{A}}{j}\right) .
\end{aligned}
$$

Hence

$$
\begin{aligned}
\sum_{j=1}^{N} p_{j} & \leq 2^{1 / \alpha} c \sum_{j=1}^{N} j^{-1 / \alpha}+\frac{2^{2 / \alpha}}{n t} \sum_{j=1}^{N} \log ^{1 / \alpha}\left(\frac{n \sqrt{A}}{j}\right) \\
& \leq 2^{1 / \alpha} c \frac{1}{1-\alpha}+\frac{2^{2 / \alpha}}{n t} n \sqrt{A} \Gamma\left(1+\frac{1}{\alpha}\right) .
\end{aligned}
$$

For $c=(1-\alpha) /\left(2^{1 / \alpha+1}\right)$ and $t \geq \sqrt{A} 4^{1 / \alpha} 2 \Gamma(1+1 / \alpha)$ we therefore have $\sum_{j=1}^{N} p_{j} \leq 1$. For $t$ satisfying the above inequality we obtain

$$
\begin{aligned}
\mathbf{P}\left(\frac{1}{n} \sum_{j \leq n} X_{j}>t\right) & \leq 2 \sum_{j \leq N} \exp \left(-c^{\alpha} n^{\alpha} t^{\alpha}\right) \leq 2 N \exp -(c n t)^{\alpha} \\
& =\exp \left(\log 2 N-(c n t)^{\alpha}\right)
\end{aligned}
$$

Since

$$
\begin{aligned}
(c t)^{\alpha} & \geq\left(\frac{1-\alpha}{2^{1 / \alpha+1}} \sqrt{A} 2^{2 / \alpha+1} \Gamma\left(1+\frac{1}{\alpha}\right)\right)^{\alpha} \\
& =2\left((1-\alpha) \Gamma\left(1+\frac{1}{\alpha}\right)\right)^{\alpha} A^{\alpha / 2}=: 2 D(\alpha),
\end{aligned}
$$

we get for values of $n$ with $\log (n+1) \leq\left(\frac{c}{2} n t\right)^{\alpha}$ (in particular, whenever $n \geq$ $\left.4(\alpha D(\alpha))^{-2 / \alpha}\right)$ the estimate

$$
\mathbf{P}\left(\frac{1}{n} \sum_{j \leq n} X_{j}>t\right) \leq \exp -\left(\frac{c}{2} n t\right)^{\alpha} .
$$

Theorem. Let $0<\alpha<1$, and let $X$ be a nonnegative random variable such that $\mathbf{E} \exp \left(X^{\alpha}\right)=A<\infty$. If $\left(X_{j}\right)_{j=1}^{n}$ are independent copies of $X$, then for

$$
t \geq \sqrt{A} 2^{2 / \alpha+1} \Gamma\left(1+\frac{1}{\alpha}\right) \text { and } n \geq \frac{4}{A}\left(\alpha^{1 / \alpha}(1-\alpha) \Gamma\left(1+\frac{1}{\alpha}\right)\right)^{-2}
$$


1162

M. SCHMUCKENSCHLAEGER

we have

$$
\mathbf{P}\left(\frac{1}{n} \sum_{j \leq n} X_{j}>t\right) \leq \exp -\left(\frac{1-\alpha}{2^{1 / \alpha+2}} n t\right)^{\alpha} .
$$

In terms of the Orlicz-norm $N_{\alpha}$ associated with the function $\exp \left(t^{\alpha}\right)-1$, ie.,

$$
N_{\alpha}(X):=\inf \left\{l>0: E \exp \left(\frac{|X|}{l}\right)^{\alpha} \leq 2\right\}
$$

we get the following

Corollary. Let $0<\alpha<1$, and let $X$ be a nonnegative random variable such that $N_{\alpha}(X)<\infty$. Then for

$$
t \geq 2^{2 / \alpha+3 / 2} \Gamma\left(1+\frac{1}{\alpha}\right) N_{\alpha}(X) \text { and } n \geq 2\left(\alpha^{1 / \alpha}(1-\alpha) \Gamma\left(1+\frac{1}{\alpha}\right)\right)^{-2}
$$

we have

$$
\mathbf{P}\left(\frac{1}{n} \sum_{j \leq n} X_{j}>t\right) \leq \exp -\left(\frac{1-\alpha}{2^{1 / \alpha+2}} n \frac{t}{N_{\alpha}(X)}\right)^{\alpha} .
$$

The result is optimal, as can be shown by choosing for $X$ a random variable with density function

$$
c_{\alpha} \exp \left(-t^{\alpha}\right), \quad c_{\alpha}=\Gamma\left(1+\frac{1}{\alpha}\right)^{-1} .
$$

In this case we conclude that

$$
\begin{aligned}
\mathbf{P}(X>x) & =\frac{c_{\alpha}}{\alpha x^{\alpha-1}} \int_{x}^{\infty} \alpha x^{\alpha-1} \exp -t^{\alpha} d t \\
& \geq \frac{c_{\alpha}}{\alpha x^{\alpha-1}} \int_{x}^{\infty} \alpha t^{\alpha-1} \exp -t^{\alpha} d t=\frac{c_{\alpha}}{\alpha} x^{1-\alpha} \exp \left(-x^{\alpha}\right) \\
& \geq \frac{c_{\alpha}}{\alpha} \exp \left(-x^{\alpha}\right) \quad \text { for } x \geq 1
\end{aligned}
$$

Thus

$$
\mathbf{P}\left(\sum^{n} X_{i}>n t\right) \geq P(X>n t) \geq \frac{c_{\alpha}}{\alpha} \exp -(n t)^{\alpha} .
$$

Remark. It is easy to get an estimate for

$$
\mathbf{P}\left(\frac{1}{n} \sum_{j \leq n} X_{j}<t E\right) \quad(t \leq 1, E:=\mathbf{E} X) .
$$

For $M>0$ we clearly have for $X_{j}^{\prime}=X_{j} I_{\left[X_{j} \leq M\right]}$

$$
\mathbf{P}\left(\frac{1}{n} \sum_{j \leq n} X_{j}<t E\right) \leq \mathbf{P}\left(\frac{1}{n} \sum X_{j}^{\prime}<t E\right) .
$$


Since $\left\|X_{j}^{\prime}\right\|_{\infty} \leq M$, the Bernstein inequality yields for $\mathbf{E} X^{\prime}-t E \geq 0$ and some constant $c_{1}$

$$
\mathbf{P}\left(\frac{1}{n} \sum_{j \leq n} X_{j}<t E\right) \leq \exp -\left(c_{1} n\left(\frac{\mathbf{E} X^{\prime}-t E}{M}\right)^{2}\right) .
$$

Since

$$
\mathbf{E} X I_{[X>M]} \leq \mathbf{E} X^{2} M^{-1}=\frac{1}{M} \mathbf{E} X^{2},
$$

we get for $M=2\|X\|_{2}^{2}\|X\|_{1}^{-1} \leq c^{\prime}(\alpha) N_{\alpha}(X)^{2} E^{-1}$ and $t \leq 1 / 2$,

$$
P\left(\frac{1}{n} \sum X_{j}<t E\right) \leq \exp \left(-c(\alpha) n\left(\frac{E}{N_{\alpha}(x)}\right)^{4}\left(\frac{1}{2}-t\right)^{2}\right) .
$$

\section{REFERENCES}

[BLM] J. Bourgain, J. Lindenstrauss, and V. D. Milman, Minkowski sums and symmetrizations, Lecture Notes in Math., vol. 1317, Springer, New York, 1986/1987.

[H] W. Hoeffding, Probability inequalities for sums of bounded random variables, J. Amer. Statist. Assoc. 58 (1963), 13-20.

[MS] V. D. Milman and G. Schechtman, Asymtotic theory of finite dimensional normed spaces, Lecture Notes in Math., vol. 1200, Springer, New York, 1986.

[P] V. V. Petrov, Sums of independent random variables, Springer, New York, 1975.

[SZ] G. Schechtman and J. Zinn, On the volume of the intersection of two $L_{p}^{n}$ balls, Proc. Amer. Math. Soc. 110 (1990), 217-224.

Institut für MAthematik, J. KePler Universität, LinZ, Austria 